\title{
Flow-Through Dissolution: A Useful Tool from Discovery Phase to Preclinical Development
}

\author{
Márta Venczel1,* Tamás Sovány², Klára Pintye-Hódi², and Gabriella Ujhelyi ${ }^{1}$ \\ 1 Sanofi, 1045 Tó u. 1-5, Budapest, Hungary \\ 2 Department of Pharmaceutical Technology, University of Szeged, 6720 Eötvös u.6., Szeged, Hungary
}

e-mail: marta.venczel@sanofi.com

\section{ABSTRACT}

The flow-through dissolution technique is a well-known approach from the early 1970 s described for low-solubility BCS Class II and IV (1) active pharmaceutical ingredients (APIs) and their drug products.

This tool is suitable for evaluating and comparing APIs and formulations and for exploring special issues related to new chemical entities. The flow-through dissolution equipment (FTDE) is used mainly for research and development studies, but pharmacopeias also make it possible to develop a method for routine analysis on FTDE, and researchers can achieve significant results even when only a few milligrams of a new chemical entity is available. Furthermore, the volume of dissolution medium required is at least four times less, which is an economic advantage. The aim of this article is to emphasize the potentials of the equipment during the discovery and preclinical/preformulation phases.

KEYWORDS: Flow-through dissolution; active pharmaceutical ingredient; solubility; solubility kinetics; solubility in FaSSIF and FeSSIF solutions; dissolution.

\section{INTRODUCTION}

.

he main limitation of the classical basket- or paddletype dissolution apparatus is the sink condition requirement because there is a high risk of quickly reaching the supersaturated concentration in a typical $1-\mathrm{L}$ dissolution vessel. Furthermore, it is sometimes not possible to achieve sink conditions for active pharmaceutical ingredients (APIs) that are practically insoluble in aqueous solutions. In the past, the majority of research compounds had a relatively low molecular weight and acceptable solubility; however, the number of larger and less soluble molecules showing permeability or solubility-limited absorption has increased in recent years (2).

The open-type flow-through dissolution technique, being a dynamic system, more closely mimics the in vivo status of the body than the static-type classical paddle and basket apparatus. The dissolved API is removed and collected from the cells of the FTDE, which provides the possibility for dissolution of a new portion of the solid material, modeling absorption and elimination.

It is possible to combine spectroscopic imaging and FTDE to improve the possibilities for investigating the release of poorly soluble APIs from pharmaceutical tablets (3).

\section{MATERIALS AND METHODS}

Flow-Through Dissolution Technique

The experimental work was carried out on an open Sotax CE 7 smart-type flow-through dissolution apparatus. This type of equipment is designed for both online spectrophotometric and offline HPLC analysis. Online spectrophotometric measurement is a suitable tool for routine analysis, when the dissolution kinetics profile is known from previous measurements. The offline configuration is preferred for the discovery and preclinical/preformulation phases, when the evaluated candidates or salts have different dissolution kinetic profiles. The set flow rate was $4.0 \mathrm{~mL} / \mathrm{min}$ to ensure suitable discriminative effect among the candidates; this is the lowest flow rate recommended by the European Pharmacopoeia and the USP. The temperature of the medium was $37.0 \pm 0.5^{\circ} \mathrm{C}$. The scheme of the equipment is presented in Figure 1.

\section{Open System}

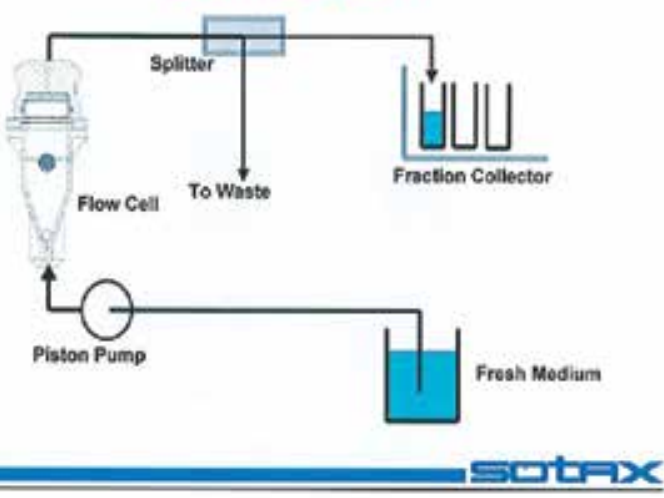

Figure 1. Open-type FTDE. 
The fraction collector is designed with $60-\mathrm{mL}$ tubes, thus the collection of all the fractions is possible with 15-min sampling intervals. If the sampling interval is increased to $30 \mathrm{~min}$, a representative sample is collected.

The end point of the studies was between 60 and 120 min, so the maximum necessary dissolution medium is between 240 and $480 \mathrm{~mL} / \mathrm{cell}$. This low volume of dissolution medium is preferred when the price of special media (e.g., fasted-state simulated intestinal fluid, FaSSIF, and fed-state simulated intestinal fluid, FeSSIF) is high because good quality lecithin and Na-taurocholate are used for solution preparation. It is feasible to mimic in vivo conditions with the media selector tool. In that case, several solutions-from acidic to neutral-flow through the same cells. Some researchers (4) have coupled FTDE with a Caco-2 cell to obtain data on the absorption behavior of the APIs.

Because open-type equipment was used for the trials, the candidates met fresh dissolution medium during the study, providing a tool for studying new molecules with low aqueous solubility.

Different types of cells are available for testing powders, granules, solid dosage formulations, and patches. The most appropriate cell for discovery and preclinical/ preformulation activities is the powder cell.

Since the particle size distribution of candidates has an impact on the solubility kinetics, it was measured by laser diffraction or by microscopic methods. The differences among particle size distributions were considered for the evaluation.

\section{Comparison of the Classical Dissolution Technique with Flow-Through Method}

During classical dissolution experiments, the dissolution medium is practically permanent (except when a medium replacement is performed during the sampling period, but this replacement is not comparable to the conditions of a flow-through dissolution technique). That is why it is challenging to ensure the sink condition requirement in case of a BCS Class II API; it is very often feasible only with surfactants. Dissolution experiments performed with a high concentration of surfactant decrease the possibility of correlation with in vivo conditions.

\section{Materials}

Some model APIs with anti-inflammatory therapeutic effect were selected.

- Model A: one chemical structure was evaluated.

- Model B: four different chemical structures were tested.
- Model C: two different salts and the base form of the API were compared and solubility in FaSSIF and FeSSIF was evaluated.

Buffer solutions were prepared according to the USP recommendations (5). The FaSSIF and FeSSIF solutions were prepared based on the description of the USP working group (6) with high quality lecithin (Lipoid E PC S, Ludwigshafen, Germany) and Na-taurocholate (Prodotti Chimici e Alimentari, Basaluzzo, Italy).

The analysis of samples was performed on an Agilent 1200 HPLC with a gradient elution method. The HPLC column used for Models A and B was a $C_{18}$ XTerra (5$\mu \mathrm{m}, 150 \times 4.6 \mathrm{~mm})$. The analysis was performed at $37^{\circ} \mathrm{C}$ with a $20-\mu \mathrm{L}$ injection volume and a flow rate of $0.8 \mathrm{~mL} /$ min. The eluent A composition was water/acetonitrile/ methane sulfonic acid (1000:25:1, v/v/v), and the eluent $B$ composition was water/acetonitrile/methane sulfonic acid (25:1000:1, v/v/v). The ratios of the $A$ and $B$ eluents of the gradient HPLC method are: 0 min (90:10); 11 min (0:100); $16 \mathrm{~min}$ (0:100); $16.5 \mathrm{~min}$ (90:10); and $23 \mathrm{~min}$ (90:10). The samples were analyzed at $220 \mathrm{~nm}$ with a UV detector. The concentrations of the standard calibration curve were 5,10 , and $25 \mu \mathrm{g} / \mathrm{mL}$.

For the Model $\mathrm{C}$ materials, the same HPLC column was used. The HPLC analysis was performed at $37{ }^{\circ} \mathrm{C}$ with a $10-\mu \mathrm{L}$ injection volume and a flow rate of $0.8 \mathrm{~mL} / \mathrm{min}$. The $A$ eluent composition was $5 \mathrm{mM} \mathrm{KH}_{2} \mathrm{PO}_{4} / 5 \mathrm{mM}$ $\mathrm{K}_{2} \mathrm{HPO}_{4}$ containing water/acetonitrile $(950: 50, \mathrm{v} / \mathrm{v})$, while the $B$ eluent was acetonitrile. The ratios of the $A$ and $B$ eluents for the gradient HPLC method are: 0 min (100:0); $10 \mathrm{~min}$ (0:100); $15 \mathrm{~min}$ (0:100); $16 \mathrm{~min}$ (100:0); and $21 \mathrm{~min}$ (100:0). The samples were analyzed at $250 \mathrm{~nm}$ with a UV detector. The concentrations of the standard calibration curve were 20,40 , and $85 \mu \mathrm{g} / \mathrm{mL}$.

\section{RESULTS AND DISCUSSION Discovery Phase}

Predicting how a drug will behave in humans before clinical testing requires a battery of sophisticated in vitro tests that complement traditional in vivo animal safety assessments (7). The quantities of promising new candidates available for early pharmaceutical evaluation are usually limited to between 10 and $20 \mathrm{mg}$ during the lead optimization process. To choose the best compounds from a biopharmaceutical point of view, physicochemical parameters such as solubility, dissolution rate, hygroscopicity, lipophilicity, $\mathrm{p} K_{\mathrm{a}}$, stability, polymorphism, and particle characteristics must be evaluated as early as possible and, above all, with the highest accuracy (8). 
For the success of the research, it is very important to initiate solubility kinetics studies in buffered and in biorelevant solutions (e.g., FaSSIF, FeSSIF) to estimate the in vivo behavior of the compounds as early as possible. Two main types of evaluation exist during the early and late discovery phases, formulation support and early biopharmaceutical evaluation of new candidates.

The particle size of the discovery candidates was less than $20 \mu \mathrm{m}$ measured by optical microscope. During the comparison of several dissolution curves of different candidates, the particle size was also measured and evaluated. This is essential based on the Noyes-Whitney equation since dissolution rate depends on both the specific surface area and the particle size distribution.

On the basis of literature data (9), the greatest effect of particle size on absorption was simulated for low dose-low solubility drugs. In general, the sensitivity of absorption to particle size decreased with increasing dose or solubility. At a solubility of $1 \mathrm{mg} / \mathrm{mL}$, particle size had practically no effect on the percentage of dose absorbed over the range of simulated doses (1-250 mg).

\section{Formulation Support: Testing of Model A Material}

Formulation development during early drug discovery and lead optimization involves several challenges including limited drug supply, the need for rapid turnaround, and limited development time. It is also desirable to develop initial formulations that will be representative of final commercial formulations (10).

The target of discovery is to screen many molecules as fast as possible. This activity can be helped if a solution formulation for the new candidates can be found. In that case, physical characterization such as determination of polymorphic forms and measurement of the particle size distribution within the suspension formulation is not necessary (11). The basic knowledge required to develop a solution formulation for new candidates is the $\mathrm{pH}$ dependent solubility profile. Measurement of the $\mathrm{pH}-$ dependent solubility properties is rather difficult if the available quantity of the candidate is only $10 \mathrm{mg}$. Flowthrough dissolution is a very good tool in that case, since there is a possibility of initiating the solubility kinetics study from $1 \mathrm{mg}$ API per powder cell.

The $\mathrm{pH}$-dependent solubility profile and $\mathrm{pH}$ range requirement of the administration route determine the pharmaceutical possibilities of the formulators. In the case of Model A material, the pH-dependent solubility profiles were measured at three different $\mathrm{pH}$ levels of 1.2, 4.5, and 7.2. The cumulative flow-through dissolution curves are presented in Figure 2. The curves show that the dissolution profiles of Model A material are faster and better at $\mathrm{pH} 1.2$ and 4.5, and the compound has very low solubility at $\mathrm{pH}$ 7.2. This low solubility at $\mathrm{pH} 7.2$ is a pharmaceutical challenge because a neutral $\mathrm{pH}$ was requested by pharmacologists for the planned nasal administration route. The formulation issue was solved with a low quantity of surfactant. The tolerability of the formulation was tested on an animal model.

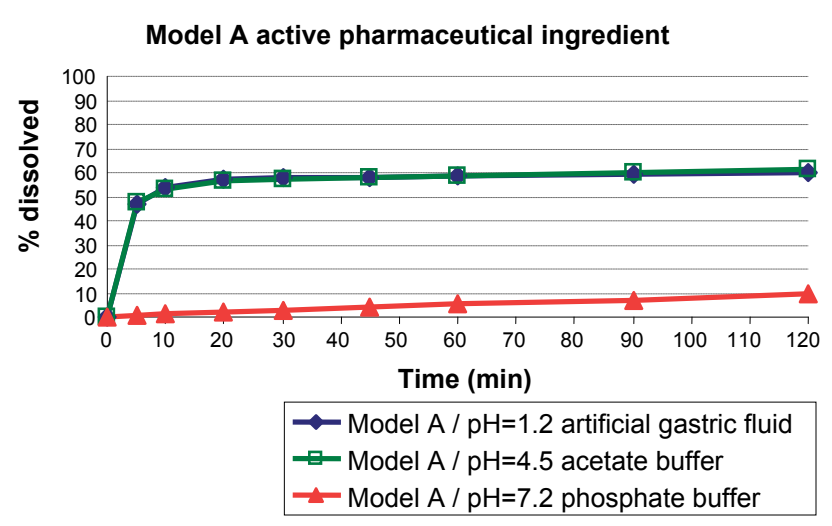

Figure 2. Cumulative dissolution curves of Model A material.

\section{Biopharmaceutical Evaluation of Discovery Model Material: Testing of Model B Material}

The objective was to select the lead candidate, not only from biological efficacy and pharmacokinetics results but also from a biopharmaceutical assessment. This example illustrates that collaboration among chemists, biologists, pharmacologists, and pharmacists as early as possible is important to identify insoluble chemical scaffolds.

Even though solubility can be estimated from the computation of the effect of each functional group individually, an exact evaluation of the solubility of the complete chemical structure in an in vitro kinetics test at $37^{\circ} \mathrm{C}$ was performed. These conditions are preferred to equilibrium solubility after 16 or $24 \mathrm{~h}$, which has less pharmaceutical relevance. On the basis of chemical structure and $\mathrm{p} K_{\mathrm{a}}$ results (Figure 3 ), the best solubility kinetic profile was expected from Structure IV at pH 1.2 in artificial gastric fluid. However, the fastest dissolution kinetics was measured for the Structure I. Structures III and IV showed interesting results with similar dissolution kinetics under acidic and almost neutral conditions as well.

The calculated or measured $\mathrm{p} K_{\mathrm{a}}$ values are available for candidates that can be ionized. From those values the possible absorption site of the candidates within the gastrointestinal tract can be evaluated. The main targets of the early biopharmaceutical evaluation are to measure the pH-dependent solubility profiles of the leads or scaffold 


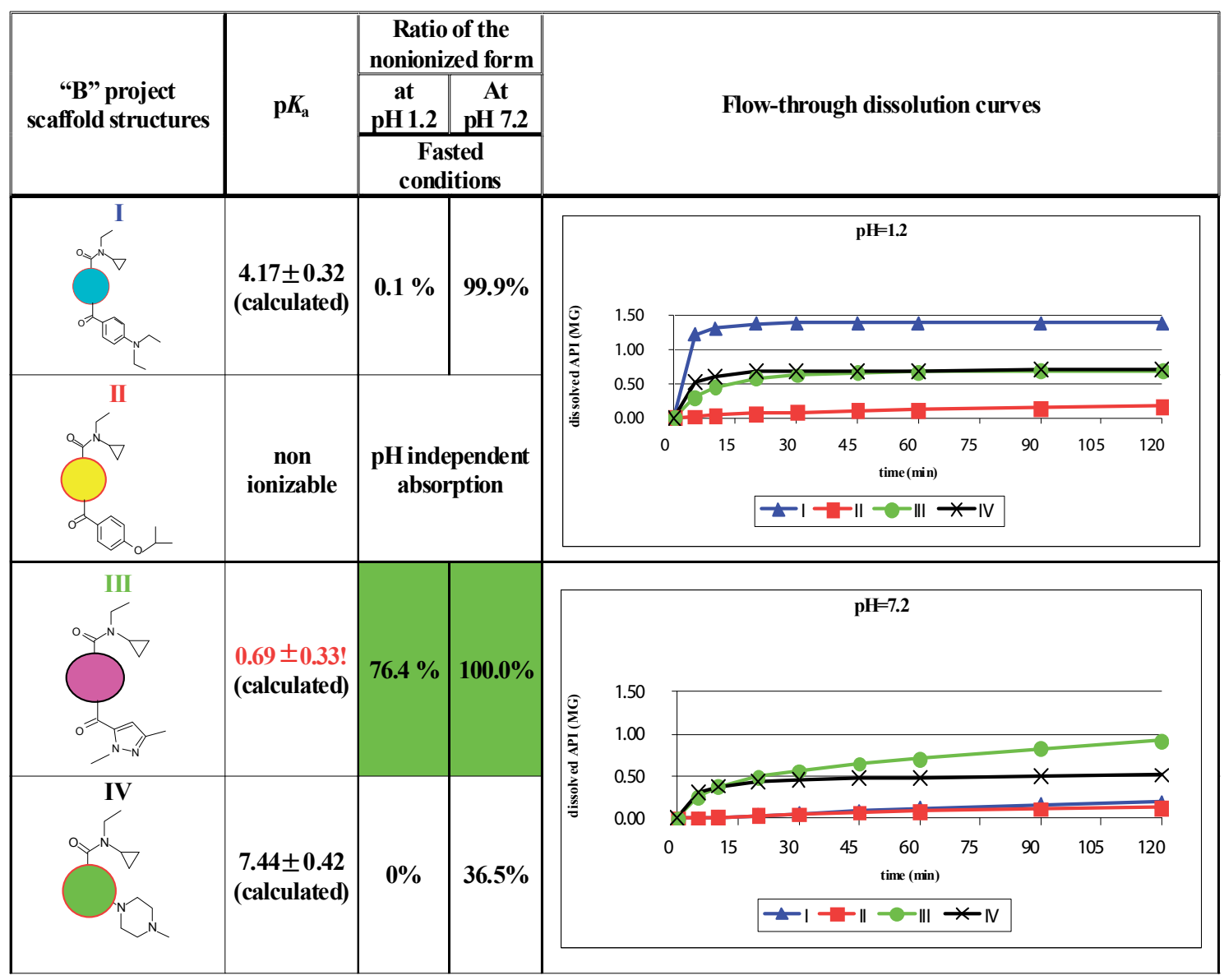

Figure 3. Selection of the best scaffold structure from biopharmaceutical point of view.

structures and to support the candidates that have better solubility properties on the estimated absorption site. The different chemical structures of Model B were evaluated on the basis of these considerations. The cumulative solubility curves (Figure 3) show that Model B Structure III has appropriate solubility at both $\mathrm{pH} 1.2$ and 7.2, which is promising because the candidate can be found in a nonionized form (suitable for absorption) at pH 7.2 under fasted conditions. Model B Structure I has an excellent solubility at a pH of 1.2 , but from pharmaceutical point of view, this form cannot be used because it can be found $99.9 \%$ in ionized form.

Structure II has $\mathrm{pH}$-independent absorption, but its solubility is rather low at both $\mathrm{pH} 1.2$ and 7.2, which is why this structure is not proposed for further development.

Structure IV has moderate and nearly similar solubility properties at $\mathrm{pH} 1.2$ and 7.2 ; however, $36.5 \%$ of the candidate can be found in a nonionized form at $\mathrm{pH}$ 7.2 according to the calculated $\mathrm{p} K_{\mathrm{a}}$ value. Because of the above mentioned facts, Structure IV has not been proposed for further development.
The flow-through dissolution technique is an excellent tool because the evaluation was available within a short time and it gave a good feedback to chemists and pharmacologists as well.

According to this approach, the new supported candidates were the ones that have acceptable aqueous solubility on the estimated place of the absorption. This is also very important from preclinical development point of view, since the costs can be reduced if, for example, particle size decrease can be omitted on the basis of the acceptable aqueous solubility property of the candidate.

\section{Preclinical Phase: Testing of Model C Material}

Higher quantities of the selected APIs were available for several preclinical activities such as pharmaceutical evaluation of several salts versus base and formulations.

\section{Comparison of Several Salts}

The flow-through dissolution study was performed on two salts (fumarate and disulfate) and the base form of the Model C. The results are presented in Figure 4. 


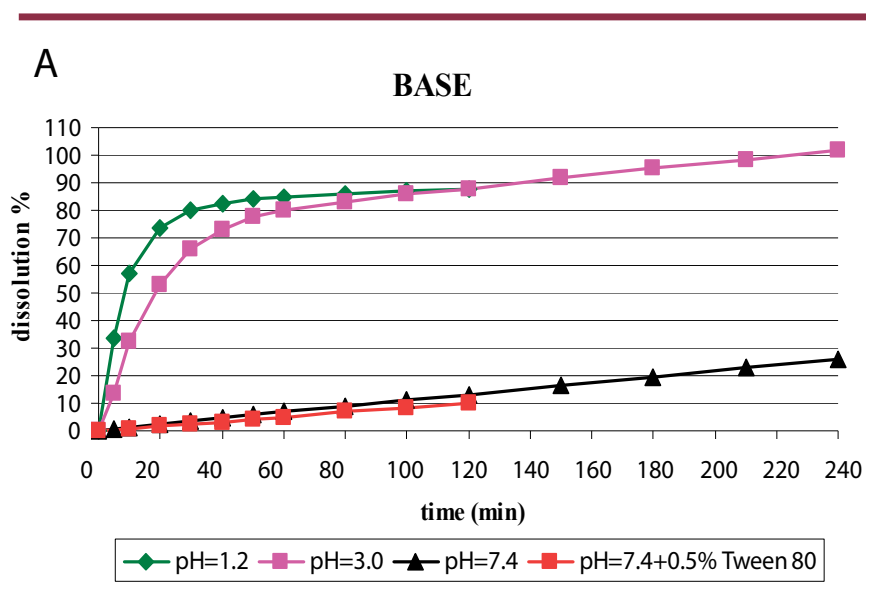

B

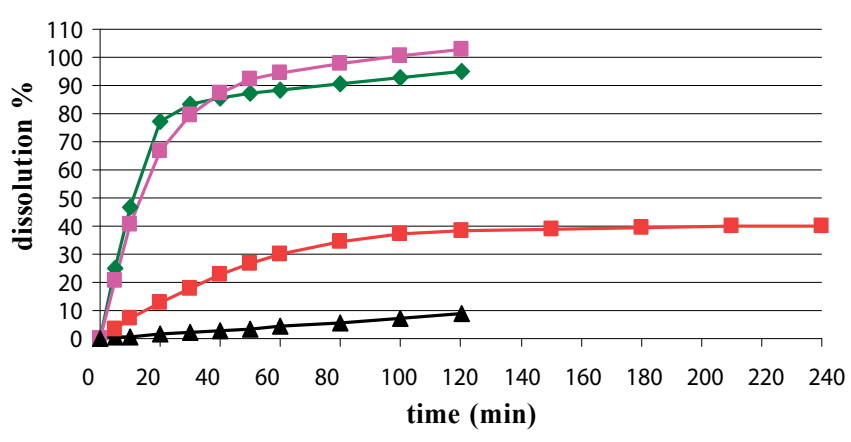

$-\mathrm{pH}=1.2-\mathrm{pH}=3.0 \multimap \mathrm{pH}=7.4-\mathrm{cH}=7.4+0.5 \%$ Tween 80

C

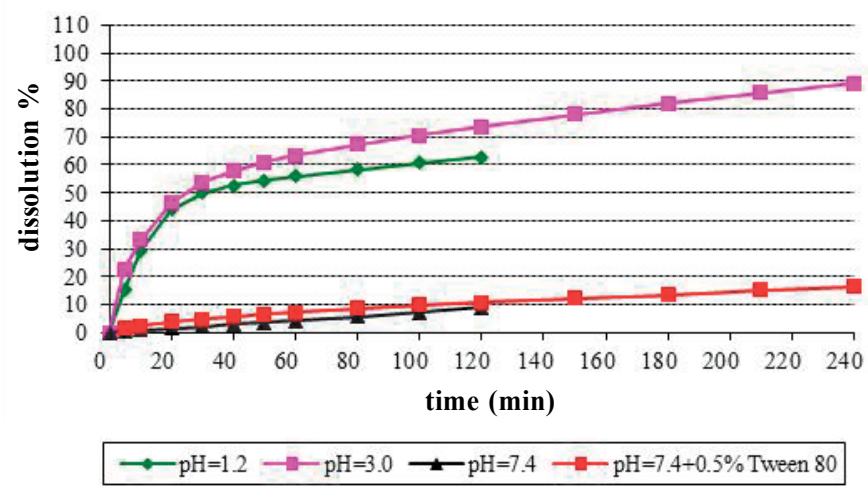

Figure 4. Comparative flow-through dissolution curves of Model C material: (A) base, (B) fumerate salt, and (C) disulfate salt.

The dissolution study was performed at $\mathrm{pH} 1.2,3.0$, and 7.4 and at $\mathrm{pH} 7.4$ with $0.5 \%$ Tween 80 . The flow-through dissolution curves show that the dissolution behavior of the two salt forms and the base form is similar (decreasing solubility from $\mathrm{pH} 1.2$ to 7.2). However, the fumarate salt has the best dissolution rate at $\mathrm{pH} 7.4$ when Tween 80 was added to the dissolution medium. This fact was used during formulation development of the fumarate salt of Model C.
Flow-Through Dissolution Study in FaSSIF and FeSSIF Solutions

The food effect prediction for Model $C$ as a fumarate salt was performed on the open-type FTDE. The most frequently used media are the fasted- and fed-state simulated small intestinal fluids (FaSSIF and FeSSIF) developed by Galia et al. (12).

The dissolution results, the calculated reaction speed constants, and the evaluation of the kinetics of the dissolution process are summarized in Table 1 . The evaluation of the dissolution kinetics was done by calculation.

\section{Evaluation of the Kinetic Order of Model C Material}

After several minutes of lag time, a clear first-order kinetics was confirmed for the flow-through dissolution in the FeSSIF medium ( $r=0.9889$ ). But the kinetics in the FaSSIF medium correlates as first-order between 30 and $60 \mathrm{~min}$ dissolution time and is pseudo first-order from $90 \mathrm{~min}$ dissolution time $(r=0.9551)$. According to the results in Table 1, the higher lecithin and Na-taurocholate contents of the FeSSIF medium ensure first-order kinetics of the Model $\mathrm{C}$ material, but in the case of the FaSSIF medium, the dissolution of the API is limited in FaSSIF medium for a 90-min dissolution time.

\section{Evaluation of the Food Effect of Model C Material}

A 1.7 times greater absorption (average of FeSSIF/FaSSIF ratio) is expected based on the in vitro flow-through dissolution results after a high fat breakfast, which means there is a slight risk for food effect. The FeSSIF/FaSSIF ratio measurement is standard during preformulation studies, but if the ratio is based on equilibrium solubility or on classical dissolution measurements, there is a high risk for much higher differences during clinical studies because classical approaches do not approximate the dynamic circumstances of the human body. Therefore, FTDE is proposed to measure the FeSSIF/FaSSIF ratio.

\section{CONCLUSION}

The flow-through dissolution technique is an excellent tool for evaluating several candidates in both the discovery and preclinical phases, in particular when low quantities of compound are available for pharmaceutical evaluation. This technique is able to support the development of a discriminative dissolution method, even if it is unfeasible with a classical dissolution approach in $500 \mathrm{~mL}$ or 1000 $\mathrm{mL}$ of dissolution medium.

This approach provides the possibility for preformulation experts to attain a pharmaceutical understanding of the molecules as early as possible. A basic knowledge of the 
Table 1. FaSSIF, FeSSIF Flow-Through Dissolution Results of Model C Material

\begin{tabular}{|c|c|c|c|c|c|c|}
\hline \multicolumn{7}{|c|}{ Dissolution medium FaSSIF, $C_{0}=1 \mathrm{mg}$} \\
\hline \multirow[b]{2}{*}{$\begin{array}{l}\text { Time } \\
(\mathrm{min})\end{array}$} & \multirow[b]{2}{*}{$\begin{array}{l}\text { Dissolved } \\
\text { (\%) }\end{array}$} & \multirow[b]{2}{*}{$\begin{array}{l}\text { Dissolved } \\
\text { (mg) }\end{array}$} & \multirow[b]{2}{*}{$\begin{array}{c}\text { Remaining } \\
\mathrm{mg}(\mathrm{C})\end{array}$} & \multicolumn{2}{|c|}{ Reaction speed constants } & \multirow[b]{2}{*}{$\begin{array}{c}\text { Evaluation } \\
\text { of the } \\
\text { kinetic }\end{array}$} \\
\hline & & & & $\begin{array}{l}\text { Zero order } \\
k=\frac{C_{0}-C}{t}\end{array}$ & $\begin{array}{c}\text { First order } \\
k=\frac{2.303}{t} \log \frac{C_{0}}{C}\end{array}$ & \\
\hline 5 & 0.02 & 0.0002 & 0.9998 & 0.0040 & 0.0000 & \multirow{2}{*}{ Lag Time } \\
\hline 10 & 3.64 & 0.0364 & 0.9636 & 0.3640 & 0.0037 & \\
\hline 30 & 35.32 & 0.35323 & 0.6468 & 1.1774 & 0.0145 & \multirow{3}{*}{ First-order } \\
\hline 45 & 48.48 & 0.48477 & 0.5152 & 1.0773 & 0.0147 & \\
\hline 60 & 57.74 & 0.57743 & 0.4226 & 0.9624 & 0.0144 & \\
\hline 90 & 65.40 & 0.65397 & 0.3460 & 0.7266 & 0.0118 & \multirow{2}{*}{$\begin{array}{l}\text { Pseudo } \\
\text { first-order }\end{array}$} \\
\hline 120 & 72.98 & 0.72977 & 0.2702 & 0.6081 & 0.0109 & \\
\hline \multicolumn{7}{|c|}{ Dissolution medium FeSSIF, $C_{0}=1 \mathrm{mg}$} \\
\hline 5 & 0.04 & 0.0004 & 0.9996 & 0.0080 & 0.0001 & \multirow{2}{*}{ Lag Time } \\
\hline 10 & 9.07 & 0.0907 & 0.9093 & 0.9073 & 0.0095 & \\
\hline 30 & 63.04 & 0.6304 & 0.3696 & 2.1012 & 0.0332 & \multirow{5}{*}{ First-Order } \\
\hline 45 & 76.54 & 0.7654 & 0.2346 & 1.7010 & 0.0322 & \\
\hline 60 & 87.33 & 0.8733 & 0.1267 & 1.4555 & 0.0344 & \\
\hline 90 & 93.95 & 0.9395 & 0.0605 & 1.0439 & 0.0312 & \\
\hline 120 & 100.51 & 1.0051 & -0.0051 & 0.8376 & - & \\
\hline
\end{tabular}

$\mathrm{pH}$-dependent solubility of the API was available within a short time with an HPLC analysis, and the results made it possible to start the formulation approach.

The opened-type FTDE represents the dynamic system of the human body in a better way than the classical paddle or basket methods, which is why FTDE has a greater role during the discovery and preclinical studies, in particular for BCS Class II and IV candidates.

\section{CONFLICT OF INTEREST}

No conflict of interest has been declared by the authors.

\section{REFERENCES}

1. Löbenberg, R; Amidon, G. L. Modern bioavailability, bioequivalence and biopharmaceutics classification system. New scientific approaches to international regulatory standards. Eur. J. Pharm. Biopharm. 2000, 50 (1), 3-12. DOI: 10.1016/ S0939-6411(00)00091-6.

2. Lipinski, C. A. Drug-like properties and the causes of poor solubility and poor permeability. J. Pharmacol. Toxicol. Methods 2000, 44 (1), 235-249. DOI: 10.1016/S1056-8719(00)00107-6.

3. van der Weerd, J.; Kazarian, S. G. Release of Poorly Soluble Drugs from HPMC Tablets Studied by FTIR Imaging and Flow-Through Dissolution Tests. J. Pharm. Sci. 2005, 94 (9), 2096-2109. DOI: 10.1002/jps.20428.

4. Motz, S. A.; Schaefer, U. F.; Balbach, S.; Eichinger, T.; Lehr, C.M. Permeability assessment for solid oral drug formulations based on Caco-2 monolayer in combination with flow through dissolution cell. Eur. J. Pharm. Biopharm. 2007, 66 (2), 286-295.
DOI: 10.1016/j.ejpb.2006.10.015.

5. Reagents, Indicators and Solutions. In The United States Pharmacopeia and National Formulary USP 37-NF 32; The United States Pharmacopeial Convention, Inc.: Rockville, MD, 2014.

6. Marques, M. Dissolution Media Simulating Fasted and Fed States. Dissolution Technol. 2004, 11 (2), 16. DOI: 10.14227/ DT110204P16.

7. Pritchard, J. F.; Jurima-Romet, M.; Reimer, M. L. J.; Mortimer, E.; Rolfe, B.; Cayen, M. N. Making Better Drugs: Decision Gates in Non-Clinical Drug Development. Nat. Rev. Drug Discovery 2003, 2, 542-553. DOI: $10.1038 / \mathrm{nrd1131.}$

8. Balbach, S.; Korn, C. Pharmaceutical evaluation of early development candidates "the 100-mg approach." Int. J. Pharm. 2004, 275, 1-12. DOI: 10.1016/j.ijpharm.2004.01.034.

9. Stegemann, S.; Leveiller, F.; Franchi, D.; de Jong, H.; Lindén, H. When poor solubility becomes an issue: From early stage to proof of concept. Eur. J. Pharm. Sci. 2007, 31 (5), 249-261. DOI: 10.1016/j.ejps.2007.05.110.

10. Chaubal, M. V. Application of formulation technologies in lead candidate selection and optimization. Drug Discovery Today 2004, 9 (14), 603-609. DOI:10.1016/S1359-6446(04)03171-X.

11. Maas, J.; Kamm, W.; Hauck, G. An integrated early formulation strategy-From hit evaluation to preclinical candidate profiling. Eur. J. Pharm. Biopharm. 2007, 66 (1), 1-10. DOI: 10.1016/j. ejpb.2006.09.011.

12. Galia, E.; Nicolaides, E.; Hörter, D.; Löbenberg, R.; Reppas, C.; Dressman, J. B. Evaluation of Various Dissolution Media for Predicting In Vivo Performance of Class I and II Drugs. Pharm. Res. 1998, 15 (5), 698-705. DOI: 10.1023/A:1011910801212. 